\title{
Analysis of Traffic Flow Equilibrium in Urban Road Network Based on Floating Vehicle Data
}

\author{
Yi Yü, Hui Gong ${ }^{2}$, Xianglun $\mathrm{Mo}^{2}$ \\ ${ }^{1}$ Shanghai Jiuxuan Engineering Technology Co, Ltd, 201000, Shanghai, China \\ ${ }^{2}$ China University of Mining and Technology, School of mines, 221116, China
}

\begin{abstract}
Based on the floating vehicle data, this paper analyzes the equilibrium of urban road network traffic flow. This can guide traffic flow distribution and provide reliable basis for traffic control. This paper starts with the quantitative analysis of the traffic network equilibrium, on the basis of verifying the validity of the floating car data, divides the urban road network into regions, and constructs an analysis model of the traffic flow equilibrium of the urban road network. The urban road traffic distribution model is constructed in accordance with the number of road traffic segments. On this basis, gini coefficient index is introduced to judge the road network flow balance, which is used to analyze the balance of each sub-region. By means of traffic guidance, signal control and other traffic control means, the traffic flow in each sub-area is balanced, and the traffic flow in the whole road network becomes balanced.
\end{abstract}

\section{Introduction}

How to improve the use of the road network under present conditions has become the focus of urban traffic research. The scientific organization, management and control of traffic has become an important trend in solving the problem of urban traffic congestion. The essence of unbalanced traffic flow is the deviation between the actual traffic volume of each function and level road in the road network and the optimal road allocation result [1]. In order to distribute the traffic demand on the road network to the traffic network according to certain routing principles, it is necessary to use the floating car data to analyze the traffic flow balance of the road network according to the actual traffic conditions. Not only can the traffic stream be assigned, but also provide a reliable basis for traffic control. Walinchus in the United States first proposed the concept of traffic control sub-areas in the road network in 1971, dividing a complex and huge road network into several independent sub-areas according to certain principles and indicators. In the research process of the division of urban road network control sub-areas, most scholars regard the intersection correlation as the main basis for the division of the control area of the area, which has produced a variety of division methods. Existing control cell division methods include spectral clustering algorithm, fuzzy neural network, dynamic division method of supersaturated control cells, correlation degree judgement, and hypergraphic division method [2-7].

In 1952, Wardrop proposed the notion and definition of the balance of the transportation network. In 1956 Beckmann and others used mathematical methods to establish a balance model of the transportation system based on the Wardrop definition of balance of the transportation system. Subsequently, people began to discuss and study the solution method of the equilibrium model proposed by Beckmann et al. In 1975, scholars such as LeBlanc used the Frank-Wolfe algorithm in nonlinear programming to obtain the solution steps and methods, and formed a reliable transportation network equilibrium model. These models and algorithms provided the basis for further research into the development of network traffic flow theory. In the development process of the balance theory of the transportation system, people consider and incorporate more factors affecting traffic in the actual transportation system when establishing the equilibrium network model. Including the consideration of elastic demand in traffic distribution, the equilibrium problem of traffic network under the mutual influence of two-way traffic flow, the consideration of link capacity, the mutual influence of road section traffic flow, and the dual criteria of time and cost are obtained in the traffic network equilibrium model [8].

National and international researchers have carried out numerous studies on the analysis of the equilibrium of the road network. Scholars such as Li Zhibin have analyzed the imbalance of the time and space distribution of the expressway from the micro level through travel time, travel time, and occupancy rate[9-11]; Deng Yajuan, Barthélemy M, Shen Hongfei mainly applied the complex network theory from the perspective of static topology, using node degree, betweenness, compactness, etc, to use entropy analyzed the imbalance of the road network structure [12-13]. The Gini factor is an important indicator for the assessment of resource allocation imbalance. In the field of the road network, it concentrates 
mainly on the unbalanced structure of the road network. He Yihao analysed the structure as a function of the density of the road network and the density of road transport and the inequality of the route [14].

At this point, much of the research focuses on the problem of traffic distribution, and there is little research on the analysis of urban traffic balance. Therefore, this article first deals with floating car data, filtering primarily, deleting duplicates, filling out missing values, and eliminating erroneous data to get valid data with search meaning. Next, establish an improved severity model and use social network analysis methods to divide the urban road network on the basis of road correlation. Finally, considering the route choice of travelers and the size of traffic flow, a balanced analysis model of each traffic subarea based on the Gini coefficient is established, which can guide the flow of traffic from a relatively unbalanced area to a more balanced area, not only Making full use of road resources can also provide a reliable basis for traffic distribution.

\section{Urban Road Network Division Based on Social Network Analysis}

The gravity model is widely used in the research of urban spatial connections, population flow, urban economics, and trade connections [15-17]. There are many influential factors in the urban highway system that are difficult to measure directly. Therefore, this paper uses the gravity model to analyze the spatial connections between roads. Because the traditional gravitational model cannot accurately reflect the attenuation of the relationship between the two research objects with distance. This paper introduces the regional spatial interaction model based on the principle of maximum entropy proposed by A G Wilson, as shown in equation (1).

$$
\begin{gathered}
T_{i j}=K Q_{i} D_{j} f\left(C_{i j}\right) \\
f\left(C_{i j}\right)=e^{-\beta r_{i j}}
\end{gathered}
$$

In the formula, $T_{i j}$ is the effect of area $i$ on area $j$; $Q_{i}$ is the total outflow from area $i ; D_{j}$ is the total inflow from area $j ; C_{i j}$ is the attenuation function, indicating that the relationship between the two changes with distance Exponential attenuation; $r_{i j}$ represents the distance between regions $i$ and $j ; \beta$ is the distance attenuation factor, the larger the value, the faster the attenuation of $f\left(C_{i j}\right)$ changes with the spatial distance.

Since the traffic flow has the spatial characteristics of continuous attenuation with the extension of distance, the attenuation function is introduced to describe the attenuation degree of road interaction, so the spatial connection strength between roads is measured as follows:

$$
F_{i j}=\frac{N_{i j}}{N_{i j}+N_{j i}} Q_{i j} Q_{j i} e^{-\beta d}
$$

In the formula, $F_{i j}$ represents the spatial connection strength between road $i$ and road $j ; N_{i j}$ and $N_{j i}$ respectively represent the number of road lanes in the direction of road $j$ and road $j$ toward road $i ; Q_{i j}$ and $Q_{j i}$ respectively represent the traffic volume from road $i$ to road $j$ and from road $j$ to road $i$; $\mathrm{d}$ represents the length of road $i ; \beta$ represents the attenuation factor between road $i$ and road $j$, Reflects the decay speed of the link between roads.

The urban road network may be divided into several subregions according to the proximity of the road interconnection. The principle of zoning in this article is that the internal connections of each subregion are more closely linked than the roads outside the subregions. Therefore, this article adopts " On the basis of "subgroup internal and external relations", the method of condensing subgroups analyzes the spatial connections between urban road networks, and divides the urban road network into several traffic sub-areas.

The block pattern method is a method for partitioning each point based on structural information. When you build the block pattern, you must use the CONCOR program in the UCINET software. CONCOR is convergent correlation. It is to repeatedly calculate the correlation coefficient between each row (or column) in a matrix (when the matrix contains the previously calculated correlation coefficient), the final result will be a correlation consisting of only 1 and -1 in the coefficient matrix. After multiple iterative calculations, CONCOR uses tree diagrams to express the degree of structural equivalence between the various locations, and marks the network members belonging to each location. The object of analysis of CONCOR is the correlation coefficient matrix, which contains the Pearson product distance coefficient, which is used to measure the similarity between player pairs. When using CONCOR for analysis, in the final output, there are preferably more than 3 players in each district. The CONCOR method can also directly analyze the multi-relational data and the multivalued relationship matrix [18].

\section{Analysis of traffic flow equilibrium in urban road network}

At present, there are mainly three commonly used calculation methods for the Gini coefficient: geometrical calculation method, indirect adjustment method and curve adjustment method. This paper adopts the curve adjustment method to analyse the balance of urban road network traffic. The specific steps are as follows:

Step1: Sort the flow betweenness value $\mathrm{fb}_{\mathrm{n}}$ of the same divided area from small to large $\mathrm{fb}(1)<\mathrm{fb}(2)<$ $\cdots<\mathrm{fb}(\mathrm{n})$.

Section flow betweenness: the ratio of the traffic flow on all paths passing through section $e_{n}$ in the road network to the sum of all traffic flows in the area. The greater the traffic between the road segment, the greater the transport network undertaken by the road segment.

$$
f b_{n}=\frac{\sum_{m \in r M} \sum_{k \in K} \tau^{k} q^{k} p_{m}^{k} \delta_{m}^{k}\left(e_{n}\right)}{\sum_{m \in M} \sum_{k \in K} \tau^{k} q^{k}}
$$


Among them, $\mathrm{fb}_{\mathrm{n}}$ is the flow betweenness of road section $\mathrm{n} ; \tau^{\mathrm{k}}$ is the conversion factor of the $\mathrm{k}$-th car model into a standard car model.

$p_{m}^{k}$ is the probability that the $k$-th vehicle type chooses path $m ; q$ is the traffic volume of motor vehicles; $\delta_{m}^{k}\left(e_{n}\right)$ is if the road $e_{n}$ is on the path $m$ in the area, then $\delta_{m}^{k}\left(e_{n}\right)=1$, otherwise $\delta_{m}^{k}\left(e_{n}\right)=0 ; \theta$ is the scale factor, and the value is 1 .

Step 2: Calculate the cumulative percent of the number of road segments in the area and the cumulative percent of road flow between the two, respectively, as follows:

$$
\begin{gathered}
x_{i}=\sum_{j=1}^{i} \frac{n_{j}}{N} \\
y_{i}=\sum_{j=1}^{i} \frac{f b(j)}{\sum_{j=1}^{i} f b(j)}
\end{gathered}
$$

Among them, $\mathrm{x}_{i}$ is the cumulative percentage of the number of road sections whose flow betweenness is less than $f b(i) ; \mathrm{y}_{i}$ is the cumulative percentage of the road flow betweenness less than or equal to $f b(i) ; \mathrm{n}_{i}$ is the number of road sections whose flow betweenness is $f b(i) ; \mathrm{N}$ is the number of road sections in the road network.
Step3: Take the cumulative percentage of the number of road sections corresponding to the section flow betweenness as the horizontal axis, and use the least squares method to fit and draw the Lorentz curve with the total cumulative percentage of the road section flow betweenness as the vertical axis.

Step4: Calculate the Gini coefficient. This article is based on the Gini coefficient calculation methodology proposed by Zhang Jianhua [19].

\section{Case Analysis}

Process the data from the floating car at 18:00 on 10 December 2018 to get the average speed of each road. According to the Xuzhou National Economic and Social Development Statistical Bulletin, as of the end of 2018, there were 1,590, 100 motor vehicles in the city, of which $1,366,400$ were civilian vehicles. As of 2018, Xuzhou had about 10,600 floating vehicles. These floating vehicles consist mainly of taxis and the Internet. Car-hailing structure, from this it can be calculated that the ratio of the number of floating cars to the actual road traffic is $1: 161$, and the traffic volume of 32 roads in the study area is shown in Fig.1. The result of the calculation of the traffic density of the route using the flow-velocity-density relationship and the analysis of the relationship between the three traffic parameters shown in Fig 2.

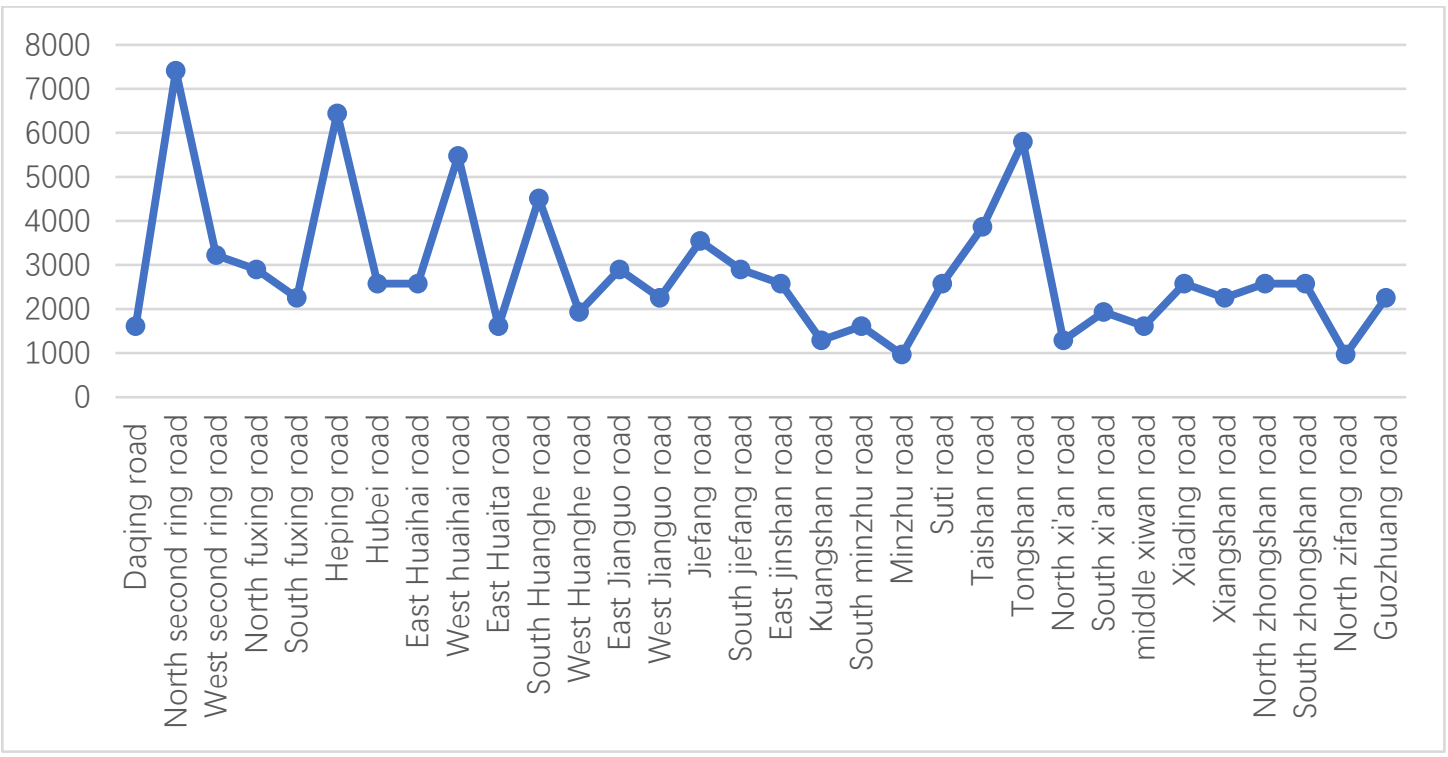

FIG. 1 Road traffic volume in the study area 


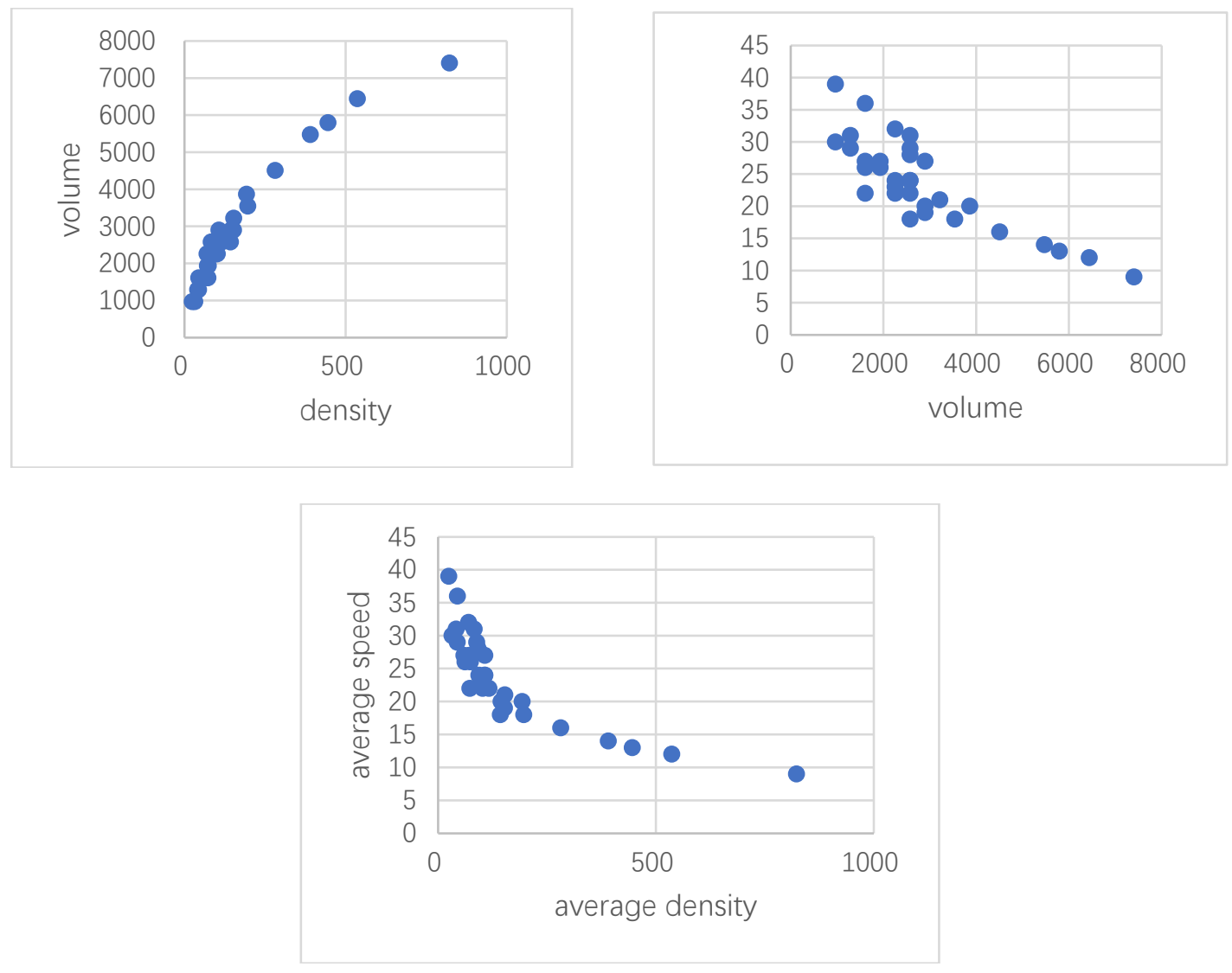

FIG.2 Relationship among the three parameters of circulation.

The following conclusions can be drawn by analysing the figure below:

West Huaihai, South Huanghe, North Second Ring, Heping, Tongshan and Jiefang highways within the study area have high traffic volumes during peak periods. The reasons are as follows: West Huaihai Road crosses the Xuzhou Central Business District and there are Important shopping malls, hospitals, banks and other places can attract more traffic; South Huanghe Road is an important road for traffic in the east-west direction of the northern part of Xuzhou City. There are many residential areas along the line, and at the same time, it shares the biggest traffic of West Huaihai Road. North Second Ring Road is an important east-west passage in the northern part of Xuzhou City, which bears a large part of the traffic volume in the northern part of the urban area; Heping Road is the core road connecting the eastern suburbs and the main areas of the urban area, and it can extend eastward to Wanda Plaza, high-speed railway station and other important locations; Tongshan Road connects National Highway G206, Huaihai Road and Jianguo Road, and is the key road from the eastern suburbs into Xuzhou City; Jiefang Road is the core road running north-south in the east of Xuzhou City Center, which connects the two Universities and the Fourth City Hospital point to the center of Xuzhou.

(2) The results correspond principally to the relationship between the three elements of circulation. Road density increases with the increase in traffic volume, road speed decreases with the increase in traffic volume, and at the same time decreases with the increase in density, indicating the fluctuation of collection. The car data has a certain amount of scientific validity.

(3) The study area is not yet overcrowded.

This paper uses Ucinet 6 software, takes the calculation results of the gravity model as input, and uses the cohesive subgroup method to analyze the closeness of the relationship between the roads in the study area. The calculation results divide the study area into four traffic sub-areas, with the second ring north road as the main one, which intersects with Daqing Road, Xiadian Road, Xiangshan Road, Second Ring West Road, and Xiyuan Middle Road to form the traffic sub-areas 1 on the north side of the study area. East Jianguo Road, Guozhuang Road, Tongshan Road, East Huaita Road, North Zifang Road, North Fuxing Road, South Fuxing Road, South Jiefang Road, and West Huanghe Road constitute the traffic sub-zone 2 on the east side of the study area; Heping Road is an east-west road that intersects South Zhongshan Road, and together with Taishan Road, South Xi'an Road, and East Huaihai Road, it forms a traffic subzone 3 in the south-central direction; Huaihai West Road and South Huanghe Road are east-west roads, and north-south roads. The intersection of North Xi'an Road and North Zhongshan Road, together with Mine Road on the west side, Mingzhu Road, Sudi Road, and Jinshan Road on the south side, and South Minzhu Road, Jiefang Road, and West Jianguo Road in the center together constitute the traffic sub-division on the west side. District 4, this zoning result basically conforms to the geographical distribution of roads in the road network, and at the same time considers the relationship between roads, which has a good zoning significance. 
This document uses Matlab 2016a to adapt to the Lorentz curves of the four circulation subzones respectively. The results of the curve fit are presented on Fig. 3, Fig.4, Fig.5, and Fig.6. The coordinate is the

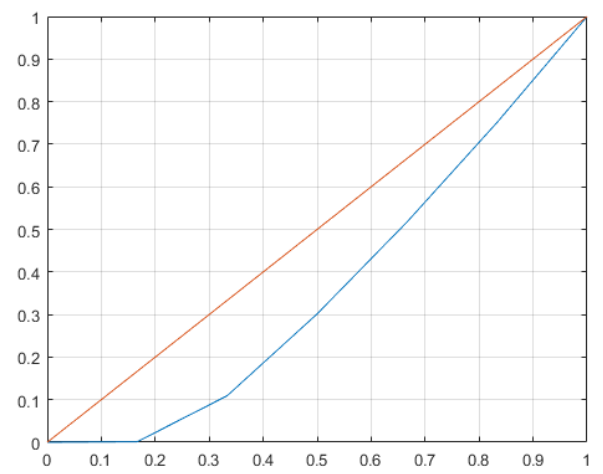

FIG. 3 Lorentz curve of traffic subregion 1

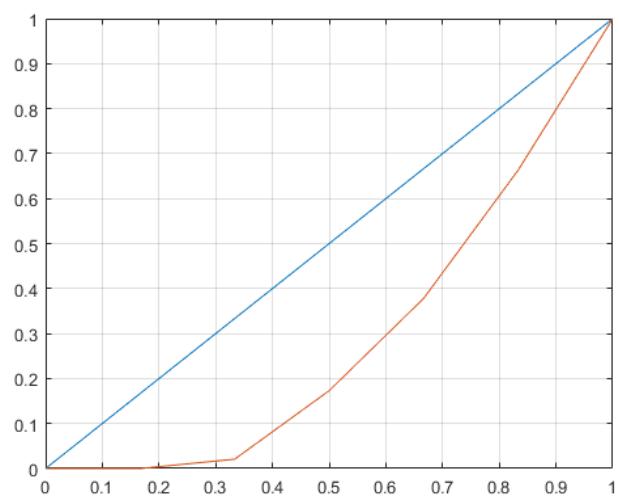

FIG. 5 Lorentz curve of traffic subregion 3 cumulative percentage of the number of road sections, and the $y$-axis is the cumulative percentage of road flow between the two.

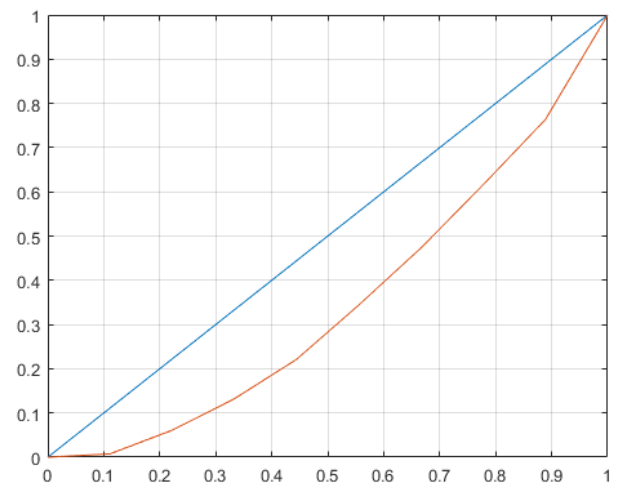

FIG. 4 Lorentz curve of traffic subregion 2

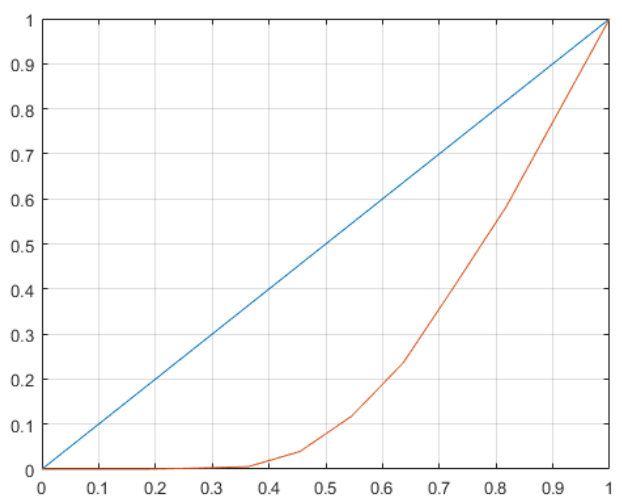

FIG. 6 Lorentz curve of traffic subregion 4

The results of the calculation of the Gini factor are presented in Table 1.

Table1.Analysis results of road network flow equilibrium based on the number of traffic segments

\begin{tabular}{cccc}
\hline Area & Lorenz curve & Gini Coefficient & Equilibrium \\
\hline sub-zone 1 & $\mathrm{y}=0.8199 \times 2+0.2319 \mathrm{x}-0.0290$ & 0.2731 & Average \\
sub-zone 2 & $\mathrm{y}=0.8550 \times 2+0.1385 \mathrm{x}-0.0082$ & 0.3068 & Smooth \\
sub-zone 3 & $\mathrm{y}=1.3333 \times 2-0.3295 \mathrm{x}+0.0026$ & 0.4216 & Variable \\
sub-zone 4 & $\mathrm{y}=1.6895 \times 2-0.7278 \mathrm{x}+0.0404$ & 0.5120 & Variable \\
\hline
\end{tabular}

Through the analysis of the calculation results, the following conclusions can be drawn:

(1) The distance between the traffic sub-areas 1 and the traffic sub-areas 2, and the Gini coefficients are 0.2731 and 0.3068 , respectively, indicating that the traffic flow distribution of the roads in the traffic sub-areas 1 and traffic sub-areas 2 is relatively even;

(2) The area enclosed by the traffic sub-zone 3 and the traffic sub-zone 4 and the absolute uniform line is relatively large, and the Gini coefficients are 0.4216 and 0.5120 , respectively, exceeding the critical value of 0.4 , indicating that the roads in the traffic sub-zone 3 and the traffic sub-zone 4 . The distribution of traffic flow is quite different. The reasons for the analysis are as follows: The main impact road of traffic flow imbalance in traffic subzone 3 is Heping Road, and the traffic flow is significantly higher than other roads in the area, resulting in an uneven distribution of regional traffic flow; traffic flow in traffic sub-zone 4 is unbalanced. The main roads affected are Zhongshan North Road and Jiefang Road. North Zhongshan Road is located in the core area of the city center. Jiefang Road is close to the city center and runs through the eastern part of the city center. Traffic on both routes is very significant and the scale has an impact.

(3) The distribution of road flow in the eastern and northern parts of the study area is relatively balanced, 
while the distribution of road flow in the central, western and southern parts is unbalanced. The specific reasons are as follows: the north is mainly residential areas, and the distribution is relatively even, and there is no important commercial center, so the distribution of traffic flow is more balanced; the east contains multiple arterial roads, which relieves the regional traffic pressure; the central part has a large number of traffic lights, The road traffic capacity is low, which conflicts with the high traffic demand; the west contains the Yunlong Lake Scenic Area, so its road network density is low, and the number of lanes is small, which is prone to traffic congestion; the southern business district and schools are more distributed It is dense and carries traffic from the southeast suburbs, so its traffic distribution is relatively uneven.

(4) The floating car can be used to guide the vehicles in the unbalanced area to achieve a more balanced state. For example, Jiefang Road in the traffic sub-zone 4 is relatively congested. The floating motor vehicle can be used to guide vehicles passing through the city center and the southern part of the city to choose Zhongshan South Road to reduce the traffic pressure on Jiefang Road, thereby alleviating the traffic imbalance in the area.

\section{Conclusion}

Traffic balance analysis is the prerequisite for a better distribution of traffic demand from the road network to the road network. Based on the correlation between roads, this paper uses social network analysis methods to divide the urban road network, and establishes a traffic flow equilibrium analysis model for the divided sub-regions. This method has a high operability and is very helpful for urban road traffic. Management and control have a degree of theoretical and practical scope.

\section{Reference}

1. T, Iryo. TRANSPORT RES B-METH 45(6), 867879, (2011)

2. H., Yin. L., Xu. Y., Cao. Journal of Transport Information and Safety, 2010(01)

3. Y. Xie, C. Li, X. Yan, H. Zhang. Journal of Guangxi University of Science and Technology, 2011(01)

4. X. Yang, W. Huang, W. Ma. Journal of Tongji University (Natural Science), 2010(10)

5. K. Lu, J. Xu, Y. Li. Journal of South China University of Technology (Natural Science Edition), 2009(07)

6. H. Duan, Z. Li, Y. Hu, Z. Zhang. Journal of Jilin University (Engineering and Technology Edition), 2009(S2)

7. Q. Yang, L Chen. Journal of Jilin University (Engineering and Technology Edition). 2006(S2)

8. H., Huang. Beijing: China Communications Press, 1994.

9. Z. Li, W. Wang, X. Li, et, al. Journal of Traffic and Transportation Engineering, 2014(14): 76-81.

10. SUN H J, WU J J, MA D, et al. APPL MATH
MODEL, 2014(38): 496-505.

11. ZOU Y J, ZHU X X, ZHANG Y L, et al. TRANSPORT RES C-EMER, 2014(43):33-49.

12. Barthélemy M. Physics Reports.2011.

13. Y. Deng, Y. Yang, G. Ma. China Journal of Highway and Transport, 2010, 23(1): 98- 104.

14. W. He, B. Fan, J. Dong, et al. Journal of Transportation Systems Engineering and Information Technology,2010, 10(6): 163-168.

15. C. Gu, H. Pang. Geographical Research.2008(01)

16. Karemera D, Oguledo V I, Davis B. Applied Economics, 2000, 32(13)

17. C. Miao, H. Wang. Geographical Research.2006(02)

18. C. M. Shuai. Chinese Rural Economy.2009(07)

19. Zhang J. Journal of Shanxi Agricultural University (Social Science Edition) 